\title{
Efeito precoce do concentrado autólogo de plaquetas sobre a osteoartrose induzida em coelhos: Níveis plasmáticos de PDGF e TGF- $\beta 1$ e seu potencial como biomarcadores ${ }^{1}$
}

\author{
Esteban Osorio-Carmona (iD ${ }^{2}$, Gilcinéa de Cássia-Santana (iD ${ }^{2}$, Jessica Alejandra Castro- \\ Varón $\mathbb{B}^{2}$, Gustavo Alves Ramalho $\mathbb{B}^{3}$, Luciana Guimarães Reis $\mathbb{( D}^{3}$, Santiago \\ Jaramillo-Colorado $\mathbb{D}^{2}$, Cleuza Maria de Faria Rezende (iD? ${ }^{2}$. \\ 2Departamento de Clínica e Cirurgia Veterinária, Universidade Federal de Minas \\ Gerais, Belo Horizonte, Brasil. \\ 3Alunos do programa de Iniciação Científica da Escola de Veterinária da UFMG, \\ Universidade Federal de Minas Gerais, Belo Horizonte, Brasil.
}

\section{freosorio105@gmail.com}

Recibido: 27 de Octubre de 2016 Aprobado: 17 de Abril de 2017 y actualizado 24 de Junio de 2017

DOI: 10.17151/vetzo.2017.11.1.8

RESUMO: O objetivo da presente pesquisa foi determinar a concentração dos fatores de crescimento PDGF e TGF- $\beta 1$ no plasma de coelhos com osteoartrose induzida pela ruptura do ligamento cruzado cranial e tratados com concentrado autólogo de plaquetas ou Ringer Lactato intra-articular. Foram empregados 14 coelhos machos, adultos, com massa corporal média de $3,0 \mathrm{~kg}$, da raça Nova Zelândia que foram divididos em dois grupos (I e II). Amostras de sangue foram coletadas antes da ruptura do ligamento cruzado cranial (momento 1), no momento da substituição do mesmo (momento 2) e duas semanas após a reparação do ligamento (momento 3) e submetidas a centrifugação para se obter o plasma. A concentração dos fatores de crescimento foi avaliada empregando-se kits de ELISA específicos para a espécie e para os fatores de crescimento. Nos coelhos do grupo concentrado autólogo de plaquetas verificou-se diferença estatisticamente significativa $(\mathrm{P}<0,05)$ nos níveis do PDGF no momento 3 quando comparados com o grupo que recebeu a solução de Ringer Lactato intraarticular. Os níveis plasmáticos do TGF- $\beta 1$ apresentaram diferença estatisticamente significativa $(\mathrm{P}<0,05)$ no grupo controle quando comparados o momento $1 \mathrm{com} o$ momento 2 e o momento 3 , porém não foi observada diferença significativa quando comparados com o grupo que recebeu o concentrado autólogo de plaquetas em nenhum dos momentos avaliados. Conclui-se, portanto, que o PDGF pode ser usado como parâmetro de avaliação precoce do efeito do concentrado autólogo de plaquetas intraarticular.

Palavras-chave: fatores de crescimento, ligamento cruzado cranial, plasma, processo articular degenerativo, terapia regenerativa 


\title{
Early effect of the autologous platelet concentrate on induced osteoarthrosis in rabbits: plasmatic levels of PDGF and TGF- $\beta 1$ and his relation as biomarkers
}

\begin{abstract}
The objective of this study was to determinate the plasmatic concentration of the PDGF and TGF- $\beta 1$ growth factors in rabbits with induced osteoarthrosis by cranial cruciate ligament rupture and treated with intra-articular autologous platelet concentrate or Ringer's lactate solution. Fourteen adult male rabbits with an average body mass of $3.0 \mathrm{~kg}$ of the New Zealand breed were divided into two groups (I and II). Blood samples were collected before cranial cruciate ligament rupture (moment 1), at substitution moment of the ligament (moment 2) and two weeks after ligament repair (moment 3 ) and centrifuged to obtain the plasma. The growth factors concentration was evaluated employing ELISA kits specifics for the specie and for growth factors. In autologous platelet concentrate group rabbits, was observed statistically significant difference $(\mathrm{P}<0.05)$ in the PDGF levels in moment 3 when compared to the group that received the intra-articular Ringer's lactate solution. The plasmatic levels of TGF- $\beta 1$ showed statistically significant difference $(\mathrm{P}<0.05)$ in the controlled group when compared moment 1 with moment 2 and moment 3 , however no significant difference was observed when compared with the group receiving the autologous concentrate platelets in any of the evaluated moments. It can be concluded, that the PDGF can be used as evaluation parameter of the early effect of the intraarticular autologous platelet concentrate.
\end{abstract}

Key words: Cranial cruciate ligament, degenerative articular process, growth factors, plasma, regenerative therapy

\section{Introdução}

A osteoartrose (OA) ou doença articular degenerativa é uma afeção multi-fatorial, progressiva, dolorosa e incapacitante, cujos tratamentos disponíveis não são satisfatórios. O diagnóstico precoce e o acompanhamento da evolução da doença e do tratamento ainda são desafios (Osorio-Carmona \& Rezende, 2014).

Dentre as modalidades de terapia celular, destaca-se a injeção de plasma rico em plaquetas, que segundo Civinini et al. (2011), despertou o interesse da comunidade científica por ser seguro, de fácil preparo e aplicação e de custo relativamente baixo. Com a introdução relativamente recente das terapias celulares em diferentes situações clínicas, surge uma possível alternativa de controle mais eficaz da OA que acomete as diferentes articulações (Patel et al., 2013). Os resultados clínicos sugerem que este produto pode ser útil no tratamento da OA, mas faltam estudos controlados que comprovem seu efeito.

Embora o CAP tenha sido empregado frequentemente como terapia para lesões do sistema musculoesquelético em veterinária (Iacopetti et al., 2012; Carmona et al., 2011; 
Saito et al., 2009) ainda não se sabe se o CAP propicia melhora temporária dos sintomas ou se esta terapia realmente tem papel importante no controle da OA.

O CAP possui um alto conteúdo de plaquetas que depois de ativadas liberam fatores de crescimento e citocinas encarregados de iniciar e modular os processos de cicatrização e regeneração tecidual (Kon et al., 2012). O CAP é uma fonte natural de fatores de crescimento tais como o fator de crescimento derivado das plaquetas (PDGF), fator de crescimento transformador $\beta 1$ (TGF- $\beta 1$ ), fator de crescimento insulínico (IGF), fator de crescimento vascular endotelial (VEGF), dentre outros, que têm funções importantes na angiogênese, mitogênese, proliferação e diferenciação celular e formação de matriz extracelular que são fundamentais para a regeneração dos tecidos e o retorno à função (Tiwari \& Bhargava, 2013; Boswell et al., 2012; Kon et al., 2012).

Há grande evidencia que existem alguns biomarcadores precoces e pré-sintomáticos da $\mathrm{OA}$, os quais se detectados, podem permitir um tratamento precoce e mais adequado. Esse ponto é chave e importante para direcionar os tratamentos da OA, uma doença caracterizada por uma prolongada fase molecular asintomática e uma fase préradiográfica que posteriormente evolui para uma fase radiográfica tardia, caracterizada por alterações estruturais dentro da articulação que levam à perda da função (Kraus et al., 2011).

O concentrado autólogo de plaquetas possui ação intra-articular, modulando o processo inflamatório e estimulando a regeneração tecidual. Adicionalmente, os fatores de crescimento regulam a presença de citocinas inflamatórias no ambiente articular responsáveis pelo avanço da osteoartrose.

O objetivo deste estudo foi avaliar o efeito precoce do concentrado autólogo de plaquetas, determinar a concentração dos fatores de crescimento PDGF e TGF- $\beta 1$ no plasma de coelhos com OA induzida experimentalmente pela ruptura do LCCr e relacionar esses fatores de crescimento como potenciais biomarcadores precoces da OA.

\section{Materiais e Métodos}

\section{Animais}

O projeto foi aprovado pela Comissão de Ética no Uso de Animais (CEUA) da Universidade Federal de Minas Gerais sob o nº 63/2014.

Foram usados 14 coelhos da raça Nova Zelândia, adultos jovens, machos com massa corporal média de $3,0 \mathrm{~kg}$. O critério de inclusão dos animais na pesquisa foi seu estado geral saudável e ausência de alterações no sistema locomotor confirmados mediante avaliação física, hemograma e bioquímica sanguínea, exame ortopédico e radiográfico nas incidências ventrodorsal e médio lateral. Os animais foram mantidos no laboratório de metabolismo animal do Departamento de Zootecnia em gaiolas individuais de tamanho $60 \mathrm{~cm} \times 60 \mathrm{~cm} \times 37 \mathrm{~cm}$, com acesso a água ad libitum e alimentação duas vezes por dia de acordo as necessidades nutricionais específicas. 
Foi feita a avaliação artroscópica da articulação FTP direita seguida da ruptura do LCCr sob visibilização artroscópica (Karl Storz., Tuttlingen, Alemanha). Decorridos 21 dias, os animais foram encaminhados para estabilização articular com auto-enxerto de fáscia lata guiado pela videoartroscopia e aplicação de CAP ou solução de Ringer Lactato (Sanobiol, São Paulo, Brasil) intra-articular.

Antes de cada intervenção foi feita terapia antibiótica com cefalotina sódica (Pharma., Aspen pharma., Rio de Janeiro, Brasil) na dose de $30 \mathrm{mg} / \mathrm{kg}$ por via IV. Após cada procedimento cirúrgico, foi feita analgesia pós-operatória empregando-se tramadol (Cristal Pharma., Belo Horizonte, Brasil) na dose de $1 \mathrm{mg} / \mathrm{kg}$ por via SC a cada 12 horas, durante três dias e terapia anti-inflamatória com meloxicam (Ourofino., Uberaba, Brasil) na dose de $0,2 \mathrm{mg} / \mathrm{kg}$ por via IM a cada 24 horas durante três dias. Depois das cirurgias foi aplicada cefalotina sódica na dose de $30 \mathrm{mg} / \mathrm{kg}$ por via IM durante 5 dias e os animais foram mantidos nas respetivas gaiolas individuais sob supervisão diária, sem imobilização do membro operado.

Após a estabilização articular, os coelhos foram divididos em dois grupos de sete animais de acordo com o tratamento. Os animais do grupo I constituíram o grupo controle e receberam $0,5 \mathrm{ml}$ intra-articular de solução Ringer Lactato e os animais do grupo II constituíram o grupo CAP e receberam $0,5 \mathrm{ml}$ intra-articular. Foram estabelecidos três momentos experimentais de acordo com os procedimentos realizados.

\section{Momento 1 (M1)}

Neste tempo, foi feita a avaliação ortopédica, goniométrica, radiográfica e ultrassonográfica dos coelhos. Foram colhidos dois $\mathrm{ml}$ de sangue para hemograma e obtenção de plasma no qual foi determinada a concentração dos fatores de crescimento PDGF e TGF $\beta 1$. Em seguida os animais foram submetidos à avaliação artroscópica da articulação FTP direita, seguida da transecção do LCCr.

\section{Momento 2 (M2)}

Após três semanas todos os animais foram submetidos às avaliações já mencionadas e encaminhados para avaliação artroscópica seguida da estabilização articular empregando-se autoenxerto de fáscia lata segundo Schawalder \& Gitterle (1989). Foram colhidos, antes da artroscopia, $2 \mathrm{ml}$ de sangue para hemograma e obtenção de plasma, no qual foi determinada a concentração dos fatores de crescimento PDGF e TGF- $\beta 1$. Nos animais que receberam o CAP foram colhidos adicionalmente $7 \mathrm{ml}$ de sangue total da veia jugular, para obter o CAP que foi injetado imediatamente após de sua obtenção.

\section{Momento 3 (M3)}

Duas semanas após todos os animais foram submetidos às avaliações mencionadas e encaminhados para avaliação artroscópica e aplicação dos respetivos tratamentos. Antes da artroscopia procedeu-se à coleta de sangue para os exames já mencionados. 


\section{Procedimentos}

\section{Anestesia}

A medicação pré-anestésica (MPA), indução e a manutenção da anestesia seguiram protocolos desenvolvidos pela equipe de anestesiologia do hospital veterinário da Escola de Veterinária da UFMG.

\section{Artroscopia \\ Ruptura do LCCr (M1)}

Foi feita uma punção no recesso supra-patelar com uma agulha hipodérmica $25 \times 7$ para coleta do líquido sinovial, seguida da dilatação da articulação com infusão de $3 \mathrm{ml}$ de Ringer Lactato. Em seguida foram feitas duas incisões cutâneas de aproximadamente 3 $\mathrm{mm}$ com lâmina de bisturi No. 22, lateral e medial ao ligamento patelar, que foram empregadas como portais de acesso para o artroscópio e os instrumentos. Seguiu-se um ponto de reparo com nylon 3-0 adjacente aos portais medial e lateral e na inserção tibial do ligamento patelar, envolvendo parcialmente o coxim adiposo infrapatelar para prevenir o deslizamento da pele e a perda dos portais artroscópicos. Utilizou-se uma lâmina de bisturi número 11 para perfurar a cápsula articular, seguindo-se a introdução da camisa artroscópica de 2,2 mm guiada por trocarte rombo. O sistema de irrigação foi conectado à bainha artroscópica e o trocarte foi substituído pelo artroscópio de 1,9 mm acoplado à câmera. Com o artroscópio posicionado na articulação foi feita a inspeção artroscópica sistemática das estruturas intra-articulares iniciando pelo recesso suprapatelar, compartimento medial, compartimento lateral, fossa intertrocantérica, ligamentos cruzados cranial e caudal e os meniscos. Após a avaliação articular e sob visibilização artroscópica procedeu-se à ruptura do $\mathrm{LCCr}$ utilizando uma pinça artroscópica de jacaré associada ou não a uma agulha hipodérmica 40x12.

\section{Reparação do LCCr (M2)}

A substituição do ligamento foi realizada conforme o descrito por Schawalder e Gitterle (1989) após avaliação artroscópica como descrito anteriormente. Foi feita uma incisão na pele iniciando no terço proximal do fêmur e estendendo-se até a fabela lateral. Posteriormente foi incisado o tecido subcutâneo até expor a fáscia lata. Com auxilio de uma tesoura Metzenbaum foi colhido um enxerto de fáscia lata em toda sua extensão e cerca de um $\mathrm{cm}$ de largura. O enxerto foi liberado no extremo proximal e manteve-se pediculado próximo à fabela lateral. Posteriormente, com auxilio de uma furadeira pneumática e com um pino de $2,5 \mathrm{~mm}$ de diâmetro foi feito um túnel tibial iniciando no ponto de inserção do LCCr no plateu da tíbia e exteriorizando medial à tuberosidade da tíbia. Com uma pinça de Deschamps o enxerto de fáscia lata foi tracionado através da fossa intercondilar e com um fio de aço $0,8 \mathrm{~mm}$ o mesmo foi passado através do túnel tibial confeccionado inicialmente. $\mathrm{O}$ enxerto de fáscia lata foi fixado na sua extremidade livre, sobre a tuberosidade da tíbia, empregando-se fio cirúrgico absorvível caprofyl 2-0 empregando em padrão simples separado. O tecido subcutâneo e a pele foram fechados de forma rotineira. Após a substituição do $\mathrm{LCCr}$ os animais foram distribuídos aleatoriamente em um dos grupos experimentais e os tratamentos foram aplicados.

\section{Obtenção das amostras do CAP e do plasma}

Foi empregado o método do tubo com centrifugação única, segundo o relatado por Silva (2012) modificando o tempo de centrifugação para 7 minutos para se obter uma maior concentração de plaquetas. Foram coletados da veia jugular dos coelhos do grupo II 7 $\mathrm{ml}$ de sangue que foram depositados em tubos de 8,5 $\mathrm{ml}$ contendo solução de ACD-A 
(BD S.A., Juiz de Fora, Brasil) como anticoagulante (citrato de trisódio; 22 g/L, ácido cítrico; $8 \mathrm{~g} / \mathrm{L}$ e dextrose; $24,5 \mathrm{~g} / \mathrm{L}$ ). As mostras foram processadas imediatamente no laboratório de patologia clínica do hospital veterinário da UFMG e realizou-se hemograma completo (Abacus Junior Vet 5., Budapest, Hungary) para avaliar os níveis basais de plaquetas no sangue total. O sangue nos tubos com ACD-A foi centrifugado a $191 \mathrm{~g}$ durante 7 minutos numa centrífuga de alta velocidade (Centrobio-80 2B, Biovera., Rio de Janeiro, Brasil). Posteriormente, com auxílio de um cateter número 20 longo e uma seringa de três $\mathrm{ml}$ foram coletados aproximadamente os primeiros $100 \mu \mathrm{L}$ da fração vermelha abaixo da interface sangue-plasma e os primeiros $900 \mu \mathrm{L}$ de plasma acima da mesma interface. Os preparados autólogos obtidos foram analisados mediante hemograma automatizado por impedância volumétrica. Cada amostra foi analisada três vezes e a média foi tomada como medida amostral para as análises estatísticas.

Os parâmetros analisados nos preparados plaquetários e no sangue total foram:

Hematócrito, contagem de plaquetas, leucócitos, valores relativos e absolutos de linfócitos, monócitos, granulócitos, volume plaquetário médio. Imediatamente antes da aplicação do CAP o mesmo foi ativado com gluconato de cálcio a 10\% (Isofarma., Ceara, Brasil) na proporção de $0,1 \mathrm{ml}$ de gluconato de cálcio por cada $\mathrm{ml}$ de CAP.

\section{Coleta e armazenamento das amostras de plasma}

Foram coletados dois ml de sangue da artéria auricular central e depositados em tubos contendo EDTA (BD S.A., Juiz de Fora, Brasil) (ácido etilenodiamino tetra-acético. $1,5 \% .2 \mathrm{mg} / \mathrm{ml}$ de sangue) e posteriormente acondicionadas em eppendorfes estéreis em duas alíquotas iguais para fazer a dosagem de TGF- $\beta 1$ e PDGF respectivamente. Após a coleta, todas as amostras foram mantidas sobre refrigeração $\left(2-8^{\circ} \mathrm{C}\right)$ até o momento do processamento.

\section{Amostras de plasma para determinação do PDGF}

A alíquota de sangue foi submetida à centrifugação (centrífuga minispin plus., São Paulo, Brasil) durante 30 minutos numa rotação de $3000 \mathrm{rpm}$, para obtenção do plasma. $\mathrm{O}$ sobrenadante dos tubos foi coletado, acondicionado em eppendorf estéril e armazenado sob refrigeração a $2-8^{\circ} \mathrm{C}$ para posterior dosagem do PDGF.

\section{Amostras de plasma para determinação do TGF- $\beta 1$}

Para obtenção do plasma procedeu-se à centrifugação das amostras de sangue durante 20 minutos à rotação de $3000 \mathrm{rpm}$. Coletou-se o sobrenadante e acondicionou-o em eppendorf. $\mathrm{O}$ plasma foi congelado e estocado a $-20^{\circ} \mathrm{C}$ até o dia do procesamento.

\section{Quantificação dos fatores de crescimento TGF- $\beta 1$ e PDGF}

A determinação das concentrações de TGF- $\beta 1$ e PDGF foram realizadas através de ensaios imuno-enzimáticos de ELISA, utilizando-se kits comerciais específicos para coelhos e de referência MBS 165545 (PDGF) MBS817924 (TGF- $\beta 1$ ), respetivamente (MyBioSource LTDA., California, USA). As amostras foram analisadas seguindo as recomendações do fabricante. O ELISA foi realizado em duplicata utilizando um leitor de ELISA multiskan FC (ThermoScientific, Massachusetts, USA).

\section{Análises estatísticas}

Para as análises estatísticas foram empregadas as seguintes provas:

- Teste de Student-Newman-Kels para avaliar variáveis pareadas; 
- Teste de Tukey para comparações de variáveis pareadas;

- Teste de regressão lineal para determinar as concentrações dos fatores de crescimento.

Os valores das médias, valores mínimos e máximos e desvio padrão obtidos dos dados observados foram comparados empregando a análise de variância ANOVA para determinar se existem diferencias significativas nas respostas aos tratamentos aplicados. O intervalo de confiança foi de 95\%, aceitando uma diferença estatística de $\mathrm{P}<0,05$.

\section{Resultados e Discussão}

\section{CAP e contagem hematológica}

Os resultados da contagem celular no sangue total e no CAP no M2 e no M3 são apresentados nas tabelas 1 e 2 como média e desvio padrão. Houve diferença significativa $(\mathrm{P}<0,05)$ entre o número de leucócitos, eritrócitos e plaquetas no sangue total e no CAP. O valor do MPV não apresentou diferença estatisticamente significativa entre os valores do sangue total e do CAP.

O protocolo proposto por Silva (2012) e modificado nesta pesquisa, foi efetivo para concentrar plaquetas de coelhos. $\mathrm{O}$ aumento em um minuto no tempo de centrifugação possibilitou obter o concentrado de plaquetas (Tab. 1 e 2) que variou entre 1,5 a 2,5 vezes os níveis basais no sangue total com média de 1,8 vezes. Entretanto não foi possível obter a concentração proposta na literatura de três a cinco vezes mais. 
Tabela 1. Valores médios (¿desvio padrão) das variáveis hematológicas no sangue total e no CAP de coelhos portadores de OA da articulação FTP (grupo II) aos 21 dias após a ruptura do $\mathrm{LCCr}(\mathrm{M} 2)$.

\begin{tabular}{|c|c|c|}
\hline Variável & $\begin{array}{l}\text { Sangue } \\
\text { inteiro }\end{array}$ & CAP \\
\hline WBC (cels $\left.\times 10^{3} / \mu \mathrm{l}\right)$ & $6,16 \pm 1,53^{3}$ & $0,39 \pm 0,23^{b}$ \\
\hline RBC (cels $\left.\times 10^{3} / \mu \mathrm{l}\right)$ & $5,28 \pm 0,85^{a}$ & $0,03 \pm 0,01^{b}$ \\
\hline $\begin{array}{c}\text { PLT } \\
\text { (fragmentos } \times 10^{3} / \mu \mathrm{l} \text { ) }\end{array}$ & $169,85 \pm 53,72^{a}$ & $312,85 \pm 78,43^{b}$ \\
\hline LYM (celsx $\left.10^{3} / \mu \mathrm{l}\right)$ & $2,70 \pm 0,35$ & --- \\
\hline LYM \% & $43,97 \pm 26,24$ & --- \\
\hline MID (cels $\left.\times 10^{3} / \mu \mathrm{I}\right)$ & $0,36 \pm 0,04$ & -- \\
\hline MID \% & $5,94 \pm 3,83$ & -- \\
\hline 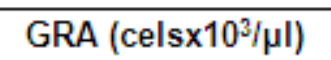 & $3,08 \pm 0,65$ & -- \\
\hline GRA \% & $50,10 \pm 25,41$ & -- \\
\hline MPV (fl) & $5,85 \pm 0,52^{a}$ & $5,88( \pm 0,31)^{a}$ \\
\hline
\end{tabular}

WBC: leucócitos; RBC: eritrócitos; HGB: hemoglobina; PLT: plaquetas; MPV: volume plaquetário médio; LYM: linfócitos; MO: monócitos; GR: granulócitos; -- = valor não detectado pelo aparelho. :\%: Médias seguidas de letras distintas diferem significativamente $(P<0,05)$. 
Tabela 2. Valores médios ( \pm desvio padrão) das variáveis hematológicas no sangue total e no CAP de coelhos portadores de OA da articulação FTP (grupo II) aos 15 dias após a substituição do $\operatorname{LCCr}(\mathrm{M} 3)$.

\begin{tabular}{|c|c|c|}
\hline Variável & Sangue inteiro & CAP \\
\hline WBC (cels $\left.\times 10^{3} / \mu \mathrm{l}\right)$ & $6,27 \pm 3,41^{a}$ & $0,30 \pm 0,11^{b}$ \\
\hline RBC (cels $\left.x 10^{3} / \mu \mathrm{l}\right)$ & $4,80 \pm 0,80^{\mathrm{a}}$ & $0,03 \pm 0,01^{b}$ \\
\hline $\begin{array}{c}\text { PLT } \\
\text { (fragmentos } \times 10^{3} / \mu \mathrm{l} \text { ) }\end{array}$ & $216,71 \pm 71,41^{a}$ & $401,62 \pm 133,99^{6}$ \\
\hline LYM (cels $\left.x 10^{3} / \mu \mathrm{l}\right)$ & $2,67 \pm 0,47$ & -- \\
\hline LYM \% & $42,70 \pm 26,91$ & --- \\
\hline MID (cels $\left.x 10^{3} / \mu \mathrm{l}\right)$ & $0,44 \pm 0,05$ & --- \\
\hline MID \% & $6,62 \pm 2,48$ & --- \\
\hline GRA (cels $\left.\times 10^{3} / \mu \mathrm{l}\right)$ & $3,16 \pm 0,65$ & -- \\
\hline GRA $\%$ & $50,54 \pm 25,37$ & -- \\
\hline MPV (fl) & $5,54 \pm 0,62^{\mathrm{a}}$ & $5,97( \pm 0,40)^{a}$ \\
\hline
\end{tabular}

Embora a literatura reporte que as concentrações ideais para se obter um efeito clínico benéfico com preparações ricas em plaquetas seja de três a cinco vezes superiores aos níveis basais, neste experimento foi considerado como CAP a fração de plasma com concentração de plaquetas entre 1,5 e 2,5 vezes os níveis basais do sangue total. Essa concentração permitiu obter efeitos clínicos favoráveis após sua aplicação intraarticular.

A concentração de leucócitos no CAP permaneceu baixa (média: 0,35 células x 10 $3 / \mu \mathrm{L}$ ), motivo pelo qual foi considerado um CAP pobre em leucócitos. O baixo número de leucócitos é considerado fundamental para se obter um concentrado autólogo de qualidade e prevenir qualquer possível reação inflamatória causada pela presença em alto número desse tipo de células (Textor, 2011). Acredita-se também, que sua presença em baixa concentração seja favorável como microbiostático (Carmona \& López, 2011; Ehrenfest et al. 2008).

Algumas vezes tem-se níveis consideráveis de leucócitos dependendo do método utilizado para obter o CAP. Esse processo baseia-se na de concentração de plaquetas no plasma, porém a centrifugação não elimina todas as outras células contidas no sangue total, sendo normal observar no CAP quantidades baixas de eritrócitos, monócitos, granulócitos e leucócitos (Wasterlain et al., 2012), assim como observado nesta pesquisa. 
Foram observadas diferenças estatisticamente significativas nas contagens dos valores hematológicos no sangue total e no CAP para os parâmetros WBC, RBC e PLT, porém não houve diferença estatisticamente significativa para os valores de MPV nos momentos analisados (Tab. 1 e 2).

São vários os fatores que interferem na funcionalidade das plaquetas e portanto, estão diretamente relacionados com a qualidade do CAP (Xie et al., 2013a; Weibrich et al., 2004; Matsuda et al., 1992). Dentre eles destacam-se o método empregado para obter o CAP e o tempo e a força da centrifugação (Tiwari \& Bhargava, 2013). O método empregado neste estudo mostrou-se eficiente para separar as diferentes células do sangue e para obter plaquetas inativas e de boa qualidade conforme o relatado por Silva et al. (2011a; 2011b) e Virchenko \& Aspenberg (2006), bem como mostrou-se eficiente na obtenção de CAP com número reduzido de leucócitos.

Múltiplas vias contribuem para a ativação das plaquetas. Durante o processo de centrifugação é possível ocorrer a ativação precoce de algumas plaquetas, o que implica em mudanças morfológicas e no tamanho das plaquetas, que podem alterar os valores do MPV (Argüelles et al., 2006). Não foi observado aumento no valor do MPV no CAP em relação ao sangue total, o que permite concluir que o método utilizado proporcionou plaquetas de qualidade e inativas. O MPV representa o tamanho médio da plaqueta e este valor pode aumentar durante a ativação plaquetária em aparelhos automatizados (Vagdatli et al., 2010; Behnke \& Forer, 1998).

Nesta pesquisa o CAP foi empregado como coadjuvante terapêutico após a reparação do LCCr para minimizar a progressão da OA, com resultados clinicamente positivos. A literatura cita seu emprego como tratamento de lesões cartilaginosas e em tendões ou como estimulador biológico para melhorar a neovascularização (Xie et al., 2013b) durante procedimentos cirúrgicos (Kon et al., 2012); o CAP tem sido usado também isoladamente ou em combinação com auto-enxertos ósseos e outros biomateriais para estimular a regeneração óssea e a reparação tecidual (Lagunas, 2006).

As plaquetas são as principais reguladoras da fase inflamatória e tem um papel fundamental na fase de proliferação e diferenciação através dos fatores de crescimento. Após uma lesão elas são ativadas e propiciam um conjunto de respostas funcionais altamente especializadas que incluem a liberação de substâncias anti e pró-inflamatórias (Intini, 2009; Harrison, 2005). O CAP é uma fonte autóloga de plaquetas e fatores de crescimento com ampla variedade de ações biológicas (Carrasco et al., 2009; Mishra et al., 2009).

Foi observada diminuição nos valores absolutos de LYM, MO e GRA no CAP em comparação com o sangue total. Isso demonstra que o método de centrifugação única permite separar eficientemente os diferentes tipos de células encontradas no sangue, facilitando assim o isolamento das plaquetas nos concentrados autólogos. Outros pesquisadores relataram que os níveis das células encontradas no concentrado autólogo de plaquetas diferem na concentração de células encontradas no sangue total, fato que poderia influenciar o efeito clínico e biológico do CAP devido à ação reguladora dos neutrófilos, macrófagos e linfócitos sobre a cicatrização (Broughton II et al., 2006a).

O aumento nos valores das plaquetas, a redução da quantidade de leucócitos e a manutenção dos valores do MPV indicam que o CAP pode ser empregado como uma 
\}

fonte segura de fatores de crescimento e citocinas que auxiliam no controle do processo inflamatório e influenciam a evolução da OA. O CAP não produz efeito deletério quando aplicado corretamente, é seguro, e elimina os riscos que traz a aplicação de medicamentos convencionais para controlar a OA (Lagunas, 2006).

\section{Fatores de crescimento}

Os resultados da determinação dos fatores de crescimento TGF- $\beta 1$ e PDGF nos momentos M1, M2 e M3 para ambos os grupos são apresentados nas tabelas 3 e 4.

Tabela 3. Valores da média e do desvio padrão dos niveis de TGF- $\beta 1$ no plasma de coelhos de ambos os grupos no M1, M2 e M3.

\begin{tabular}{|c|c|c|}
\hline \multicolumn{3}{|c|}{ Concentração plasmática do TGF- $\beta 1$} \\
\hline & Grupo controle & Grupo CAP \\
\hline M1 & $153,66 \pm 20,88^{\mathrm{BA}}$ & $157,95 \pm 12,08^{\mathrm{zA}}$ \\
\hline M2 & $187,44 \pm 24,31^{\mathrm{bA}}$ & $158,12 \pm 21,59^{\mathrm{BA}}$ \\
\hline M3 & $196,31 \pm 16,18^{\mathrm{bA}}$ & $177,22 \pm 34,41^{\mathrm{BA}}$ \\
\hline
\end{tabular}

M1: momento 1; M2: momento 2; M3: momento 3; CAP: concentrado autólogo de plaquetas.: Letras minúsculas diferentes nas colunas apresentam diferença estatisticamente significativa $(P<0,05)$. ${ }^{\star}$. Letras maiúsculas diferentes nas linhas apresentam diferença estatisticamente significativa $(P<0,05)$.

Tabela 4. Valores da média e desvio padrão dos níveis de PDGF no plasma de coelhos de ambos os grupos no M1, M2 e M3.

\begin{tabular}{|c|c|c|}
\hline \multicolumn{3}{|c|}{ Concentração plasmática do PDGF } \\
\hline $\begin{array}{c}\text { Grupo } \\
\text { controle }\end{array}$ & Grupo CAP \\
\hline M1 & $74,98 \pm 43,07^{\mathrm{BA}}$ & $78,76 \pm 15,83^{\mathrm{BA}}$ \\
\hline M2 & $\begin{array}{c}81,17 \\
( \pm 34,33)^{\mathrm{BA}}\end{array}$ & $124,26 \pm 28,30^{\mathrm{Bb} A}$ \\
\hline M3 & $93,39 \pm 26,60^{\mathrm{BA}}$ & $146,69 \pm 47,38^{\mathrm{bB}}$ \\
\hline
\end{tabular}

M1: momento 1; M2: momento 2; M3: momento 3; CAP: concentrado autólogo de plaquetas. $: 0$ : Letras minúsculas diferentes nas colunas apresentam diferença estatisticamente significativa $(P<0,05){ }^{A} \AA$ : Letras maiúsculas diferentes nas linhas apresentam diferença estatisticamente significativa $(P<0,05)$.

No grupo controle foram observadas diferenças estatisticamente significativas $(P<0,05)$ nas concentrações do TGF- $\beta 1$ entre M1 e M2 e entre M1 e M3, porém não houve diferença significativa entre M2 e M3 (Tab. 3). Enquanto no grupo CAP não se verificou diferença estatisticamente significativa em nenhum dos momentos avaliados. 
Quando comparados os grupos, também não se verificou diferença estatisticamente significativa nas concentrações do TGF- $\beta 1$ (Tab. 3). Isto sugere que há alguma influência do CAP sobre este fator de crescimento no meio articular.

As concentrações do PDGF nos diferentes tempos avaliados, não mostraram diferença estatisticamente significativa no grupo controle (Tab. 4). Entretanto no grupo CAP, ocorreu diferença estatisticamente significativa $(\mathrm{P}<0,05)$ nas concentrações do PDGF entre o M1 e o M3, sem diferença significativa entre o M1 e o M2 e entre o M2 e o M3.

$\mathrm{Na}$ comparação entre os grupos, verificou-se diferença estatisticamente significativa $(\mathrm{P}<0,05)$ na concentração do PDGF no M3 (Tab. 4).

Os níveis do PDGF no grupo CAP mostraram aumento progressivo até o M3 (Tab. 4). Este aumento pode ser devido á liberação deste fator de crescimento pelas plaquetas presentes no CAP após sua ativação e por algumas células como macrófagos e linfócitos.

Acredita-se que a aplicação de CAP intra-articular induz aumento da concentração do PDGF no plasma devido ao estímulo bioquímico dos fatores de crescimento sobre as células locais e a degranulação plaquetária. Os fatores de crescimento são proteínas pequenas secretadas no espaço intercelular e exercem papel fundamental como mediadores biológicos na regulação da migração, diferenciação, proliferação celular e equilíbrio da MEC (Sánchez et al., 2003; Kang \& Kang, 1999). Eles se unem a receptores específicos de superfície das células para posteriormente ativar um segundo mensageiro que induz uma determinada resposta no núcleo (García-Denche, 2006).

A regeneração dos tecidos moles, de feridas e de ossos dependem da ação de diferentes componentes sanguíneos como a fibrina, a fibronectina, os fatores de crescimento, dentre outros e a presença dos fatores de crescimento em níveis elevados pode alterar ou acelerar esse processo (Schwartz et al., 2011; Hakimi et al., 2010; Broughton II et al., 2006b). Existem diferentes fatores de crescimento com ampla variedade de funções, dentre as quais se podem citar a angiogênese, a mitogênese e a remodelação da MEC (Schwartz et al., 2011; Bennett \& Schultz, 1993). Portanto, espera-se algum efeito benéfico no controle das lesões articulares.

Os grânulos $\alpha$ das plaquetas contêm proteínas que influenciam a cicatrização das feridas (Flores et al., 2012) e tem sido proposto o uso de CAP para acelerar o processo de cicatrização, reduzir o processo inflamatório, estimular a capacidade de regeneração tecidual e diminuir a atividade fibroblástica, minimizando a produção de tecido cicatricial não funcional (Lynch et al., 1989). As plaquetas têm um papel fundamental no processo de reparação tecidual devido à liberação de grande quantidade de fatores de crescimento que induzem angiogênese, a proliferação e diferenciação celular, a deposição de matriz extracelular e aumentam a quimiotaxia (Carmona \& Lopez, 2011).

Os fatores de crescimento, dentre eles TGF- $\beta 1$ e o PDGF apresentam diversas funções biológicas e influenciam o meio onde são aplicados estimulando a neovascularização e a diferenciação celular (Anitua et al., 2012; Carmona et al., 2011; Smith \& Roukis, 2009; Riedel et al., 2007). Assim, o aumento do PDGF no grupo CAP pode ter favorecido um 
processo inflamatório menos acentuado, propiciando maior apoio do membro operado e proporcionando bem-estar e conforto.

Segundo Marx (2004), a liberação dos fatores de crescimento pelas plaquetas inicia-se uma hora depois da ativação e segundo Lee et al. (2012), alcança sua máxima intensidade 12 horas após, com liberação de aproximadamente $47 \%$ da totalidade desses fatores. $\mathrm{O}$ autor relata ainda que a liberação dos fatores de crescimento é mantida por aproximadamente sete dias após iniciada a ativação plaquetária. Estas afirmações conduzem a questionar o protocolo de aplicação quinzenal.

Patel et al. (2013) e Milano et al. (2010) descreveram o efeito benéfico do CAP sobre as estruturas articulares, o que favorece o uso do membro e a amplitude do movimento articular em animais acometidos pela OA.

O PDGF e o TGF- $\beta 1$ são os principais fatores de crescimento presentes no CAP e regulam o equilíbrio entre as citocinas e os fatores de crescimento para favorecer a recuperação dos tecidos lesados (Kim et al., 2008; Niu \& Rosen, 2005). Os fatores de crescimento são produzidos por outras células como os macrófagos e os linfócitos para estimular a epitelialização e a angiogênese (Lawrence \& Diegelmann, 1994). Petrigliano et al. (2006) recomenda estabelecer a concentração ideal e a quantidade de CAP a ser aplicada para se obter um resultado clinicamente aceitável.

No M3, no grupo controle, a OA se mostrou clinicamente mais acentuada. A presença do TGF- $\beta 1$ em alto nível em articulações com artrite sugere que este fator de crescimento isolado ou em associação com outras citocinas têm importante papel como biomarcador da progressão de doenças articulares. Embora este fator de crescimento tenha propriedades anti-inflamatórias ele pode também promover inflamação articular (Cheon et al., 2002), caso haja um estímulo para isto.

Algumas pesquisas têm encontrado que o TGF- $\beta 1$ pode modular a expressão e liberação de citocinas inflamatórias como o FNT- $\alpha$ e a IL-1 e pode acelerar a hipertrofia sinovial, pois esse fator de crescimento induz proliferação dos fibroblastos. A produção e atividade das metaloproteinases são reguladas pelo TGF- $\beta 1$ (Sohn et al., 2012). Associando as informações clínicas obtidas e os níveis desse fator de crescimento no plasma pode-se explicar as alterações mais acentuadas nos coelhos do grupo controle no M3.

Estimuladas pelos sinoviócitos e os condrócitos, as citocinas podem influenciar o resultado da resposta inflamatória perpetuando o processo inflamatório através das metaloproteinases liberadas pelas células (Rezende \& Campos, 2013; Bonnet \& Walsh, 2005). Algumas citocinas têm papel fundamental na inflamação articular e na degradação da cartilagem. Em pacientes com ruptura do LCCr ocorre um desequilíbrio entre as citocinas pró-inflamatórias e as citocinas anti-inflamatórias que ocasiona lesão da cartilagem e conduz á AO (Doom et al., 2008; Cameron et al., 1997).

Segundo o relatado por Baksh et al. (2013) depois da ativação e aplicação do CAP há um aumento nos níveis dos fatores de crescimento no local afetado. Nesta pesquisa observou-se no grupo CAP valores significativamente maiores do PDGF e valores menores não significativos do TGF- $\beta 1$ no M3. 
Os achados desta pesquisa evidenciam que o CAP induz um aumento nos níveis dos fatores de crescimento, principalmente o PDGF, e regula os níveis e a função do TGF$\beta 1$, modulando assim o processo inflamatório e retardando o avanço da OA. Esse efeito é evidenciado clinicamente através da melhora da amplitude de movimento articular, do alivio da dor e do uso precoce do membro depois da ruptura do LCCr, assim como evidenciado. Embora este estudo tenha avaliado o efeito inicial do CAP, é possível observar o potencial benéfico e as melhoras clínicas obtidas após seu uso, fato pelo qual é necessária uma avaliação tardia dos níveis dos fatores de crescimento e uma avaliação histológica dos tecidos envolvidos para evidenciar o efeito celular do CAP.

Finalmente, os níveis plasmáticos do PDGF e do TGF- $\beta 1$ poderiam ser usados como biomarcadores da OA por participarem ativamente da regulação e avanço da OA, sempre e quando sejam feitas todas as análises de validação e qualificação exigidos pela OARSI (Kraus et al., 2015). Um biomarcador é uma característica que pode ser objetivamente medida e avaliada como um indicador de um processo biológico normal, um processo patológico ou uma resposta farmacológica a uma intervenção terapêutica (Colburn et al., 2001).

Embora tenham sido feitos grandes avanços em relação ao diagnóstico e tratamento da $\mathrm{OA}$, as ferramentas disponíveis atualmente para diagnosticar precocemente a doença ainda são ineficazes. Os biomarcadores podem ser substancias bioquímicas, proteínas, fragmentos de DNA e RNA que podem ser inclusos em diferentes categorias (diagnóstico, prognóstico, estagio da doença, eficácia da intervenção e investigação) e obtidos de diferentes fontes (líquido sinovial, plasma, sangue) ou escalas análogas de avaliação (Kraus et al., 2011; Bauer et al., 2006).

Considerando o anterior, o PDGF e o TGF- $\beta 1$ poderiam ser inclusos em várias dessas categorias, considerando que eles podem ser obtidos de diferentes fontes (plasma, líquido sinovial) e que participam ativamente da OA. Esses fatores de crescimento estão contidos no CAP e poderiam ser indicativos da resposta ao tratamento com essa terapia. Embora essas moléculas possam ser úteis como biomarcadores, é necessário realizar pesquisas controladas e seriadas para validar e qualificar essas substancias como biomarcadores precoces da $\mathrm{OA}$, considerando adicionalmente, que os níveis plasmáticos e no líquido sinovial podem ser afetados por outras patologias sistémicas como alterações inflamatórias e até câncer (Werner \& Grose, 2003).

\section{CONCLUSÃO}

Os resultados obtidos nesta pesquisa permitem concluir que o PDGF e o TGF- $\beta 1$ podem ser usados como parâmetros de avaliação da ação do CAP intra-articular. O CAP pode ser empregado como terapia coadjuvante nas etapas iniciais da OA decorrente da ruptura do LCCr. Os fatores de crescimento avaliados nesta pesquisa poderiam ser utilizados como biomarcadores precoces da OA devido à sua participação no avanço dessa doença, fato que permitiria avaliar a progressão e o prognóstico da OA. Os fatores de crescimento podem ser utilizados como biomarcadores para avaliar à resposta ao tratamento com CAP, sempre e quando essas substâncias sejam validadas através de 
pesquisas controladas conforme às exigências internacionais, considerando as potenciais fontes de variação causadas por outros processos patológicos.

\section{Referências}

- Anitua, S.; Prado, R.; Sánchez, M. et al. Platelet-rich plasma: preparation and formulation.Operative Technique in Orthopaedic, v.22, p. 25-32. 2012.

- Argüelles, D.; Carmona, J.U.; Pastor, J. et al. Evaluation of single and double centrifugation tube methods for concentrating equine platelets. Research in Veterinary Science, v.81, p.237-245. 2006.

- Baksh, N.; Hannon, C.P.; Murawski, C.D. et al. Platelet-rich plasma in tendon models: a systematic review of basic science literature. Arthroscopy, v. 29, n. 3, p. 596-607. 2013.

- Bauer, D.C.; Hunter, D.J.; Abramson, S.B. et al. Classification of osteoarthritis biomarkers: a proposed approach. Osteoarthritis and Cartilage, v.14, p.723-727. 2006.

- Behnke, O.; Forer, A. From megakaryocytes to platelets: platelet morphogenesis takes place in the bloodstream. European Journal of Haematology, v.60, p.3-24, 1998.

- Bennett, N.T.; Schultz, G.S. Growth factors and wound healing: Biochemical properties of growth factors and their receptors. American Journal of Surgery, v.165, n.6, p.728737. 1993.

- Bonnet, C.S.; Walsh, D.A. Osteoarthritis, angiogenesis, and inflammation. Rheumatology (Oxford), v.44, n. 1, p.7-16. 2005.

- Boswell, S.G.; Cole, B.J.; Sundman, E.A. Platelet-rich plasma: a milieu of bioactive factors.Arthroscopy, v.28, n.3, p.429-439. 2012.

- Broughton II, G.; Janis, J.E.; Attinger, C.E. The basic science of wound healing. Plastic and Reconstructive Surgery, v.117, p.12S-32S, 2006a (Suppl.7).

- Broughton II, G.; Janis, J.E.; Attinger, C.E. Wound Healing: An Overview. Plastic and Reconstructive Surgery, v.117, p.1e-S-32e-S, 2006b (Suppl.7).

- Carmona, J.U.; López, C. Autologous platelet concentrates as a treatment for shoulder injury in a horse. case report. Journal of Equine Veterinary Science, v.31, n.9, p. 506510. 2011. 
\}

- Carmona, J.U.; López, C.; Giraldo, C.E. Uso de concentrados autólogos de plaquetas como terapia regenerativa de enfermedades crónicas del aparato musculoesquelético equino.Archivo de Medicina Veterinaria, v.43, n.1, p.1-10. 2011.

- Carrasco, J.; Bonete, D.; Gomar, R. Plasma rico en plaquetas vs. plasma rico em factores de crecimiento. Revista Española de Cirugía Osteoarticular, v.46, n.239, p.127-140. 2009.

- Cheon, H.; Yu, S.J.; Yoo, D.H. et al. Increased expression of pro-inflammatory cytokines and metalloproteinase-1 by TGF-b1 in synovial fibroblasts from rheumatoid arthritis and normal individuals. Clinical and Experimental Immunology, v.127, n.3, p.547-552. 2002.

- Civinini, R.; Macera, A.; Nistri, L. et al. The use of autologous blood-derived growth factors in bone regeneration. Clinical Cases Mineral Bone Metabolism, v.8, n.1, p.25$31,2011$.

- Colburn, W.A.; De Gruttola, V.G.; De Mets, D.L. et al. Biomarkers and surrogate endpoints: preferred definitions and conceptual framework. Biomarkers Definitions Working Group. Clinical Pharmacological \& Therapeutics, v.69, p. 89-95. 2001

- Ehrenfest, D.M.D.; Rasmusson, L.; Albrektsson, T. Classification of platelet concentrates: from pure platelet-rich plasma (P-PRP) to leucocyte- and platelet-rich fibrin (LPRF). Trends in Biotechnology, v. 27, n. 3, p. 158-167. 2008.

- Flores, J.R.; Gallego, M.A.P.; García-Denche, J.T. Plasma rico en plaquetas: fundamentos biológicos y aplicaciones en cirugía maxilofacial y estética facial. Revista Española de Cirugía Oral y Maxilofacial, v. 34, n. 1, p. 8-17. 2012.

- Harrison, P. Platelet function analysis. Blood Reviews, v.19, n.2, p.111-123. 2005.

- Hakimi, M.; Jungbluth, P.; Sager, M. et al. Combined use of platelet-rich plasma and autologous bone grafts in the treatment of long bone defects in mini-pigs. InjuryInternational Journal of the Care of the Injured, v.41, n.7, p. 717-723. 2010.

- Iacopetti, I.; Perazzi, A.; Ferrari, V. et al. Application of platelet-rich gel to enhance wound healing in the horse: A case report. Journal of Equine Veterinary Science, v.32, n.3, p.123-128. 2012.

- Intini, G. The use of platelet-rich plasma in bone reconstruction therapy. Biomaterials, v.30, n.28, p.4956-4966. 2009.

- Kang, H.J.; Kang, E.S. Ideal concentration of growth factors in rabbit's flexor tendon culture.Yonsei Medical Journal, v.40, n.1, p.26-29. 1999. 
- Kim, J.H; Park, C.; Park, H.M. Curative effect of autologous platelet-rich plasma on a large cutaneous lesion in a dog. Veterinary Dermatology, v.20, n.2, p. 123-126. 2008.

- Kon, E.; Filardo, G.; Di Matteo, B. et al. Platelet-rich plasma in sports medicine: new treatment for tendon and cartilage lesions. Operative Techniques in Orthopaedics, v.22, n.2, p.78-85. 2012.

- Kraus, V.B.; Blanco, F.J.; Englund, M. OARSI Clinical Trials Recommendations: Soluble biomarker assessments in clinical trials in osteoarthritis. Osteoarthritis and Cartilage, v.23, p.686-697, 2015.

- Kraus, V.B.; Burnett, B.; Coindreau, J. et al. Application of biomarkers in the development of drugs intended for the treatment of osteoarthritis. Osteoarthritis and Cartilage, v.19, p.515-542, 2011.

- Lagunas, J.G. Plasma rico en plaquetas. Revista Española de Cirugía Oral y Maxilofacial, v.28, n.2, p.89-99. 2006.

- Lawrence, W.; Diegelmann, R. Growth factors in wound healing. Clinics in Dermatology, v.12, n.1, p.157-169. 1994.

- Lee, A.J.; Chung, W.H.; Kim, D.H. et al. Anterior cruciate ligament reconstruction in a rabbit model using canine small intestinal submucosa and autologous platelet-rich plasma. Journal of Surgery Research, v.178, n.1, p.206-215. 2012.

- Lynch, S.E.; Williams, R.C.; Polson, A.M. et al. A combination of platelet derived and insulin-like growth factors enhances periodontal regeneration. Journal of Periodontology, v. 16, n. 8, p. 545-548. 1989.

- Marx, R.E. Platelet-rich plasma: evidence to support its use. Journal of Oral and Maxillofacial Surgery, v. 62, n. 4, p. 489-496. 2004.

- Matsuda, N.; Lin, W.L.; Kumar, N.M. et al. Mitogenic, chemostatic, synthetyc responses of rat periodontal ligament fibroblastic cells to polypeptide growth factors in vitro. Journal of Periodontology, v.63, n.6, p.515-25. 1992.

- Milano, G.; Sanna-Passino, E.; Deriu, L. et al. The effect of platelet rich plasma combined with microfractures on the treatment of chondral defects: an experimental study in a sheep model. Osteoarthritis and Cartilage, v.18, n.7, p.971-980. 2010.

- Mishra, A.; Woodall, J.; Vieira, M. Treatment of tendon and muscle using platelet-rich plasma. Clinics in Sports Medicine, v.28, n.1, p.113-125. 2009.

- Niu, T.; Rosen, C.J. The insulin-like growth factor-I gene and osteoporosis: a critical appraisal. Gene, v.361, p.38-56. 2005. 
- Osorio-Carmona, E.; Rezende, C.M.F. Osteoartrose: aspectos clínicos e novas perspectivas terapêuticas baseadas na terapia regenerativa. Veterinaria y Zootecnia, v.8, n.2, p.49-71. 2014.

- Patel, S.; Dhillon, M.S.; Aggarwal, S. et al. Treatment with platelet-rich plasma is more effective than placebo for knee osteoarthritis: A prospective, double-blind, randomized trial.American Journal of Sports Medicine, v.41, n.2, p.356-364. 2013.

- Petrigliano, F.P.; McAllister, D.R.; Wu, B.M. Tissue engineering for anterior cruciate ligament reconstruction: A review of current strategies. Journal of Arthroscopic Related Surgery, v.22, n.4, p.441-451. 2006.

- Rezende, M.U de.; Campos, G.C de. A osteoartrite é uma doença mecânica ou inflamatória?Revista Brasileira de Ortopedia, v.48, n.6, p.471-474. 2013.

- Riedel, K.; Riedel, F.; Goessler, U.R. et al. TGF-beta antisense therapy increases angiogenic potential in human keratinocytes in vitro. Archives of Medical Research, v.38, n.1, p.45-51. 2007.

- Saito, M.; Takahashi, K.A.; Arai, Y. et al. Intraarticular administration of platelet-rich plasma with biodegradable gelatin hydrogel microspheres prevents osteoarthritis progression in the rabbit knee. Clinical and Experimental Rheumatology, v. 27, n. 2, p. 201-207. 2009.

- Sánchez, M; Azofra, J; Anitua, E. et al. Plasma rich in growth factors to treat an articular cartilage avulsion: a case report. Medicine and Science in Sports and Exercise, v.35, n.10, p.1648-1652. 2003.

- Schawalder, P.; Gitterle, E. Eigene methoden zur operativen rekonstruktion bei rupturen des vorderen und hinteren kreuzbandes. Kleintierpraxis, v.7, p.323-330, 1989.

- Schwartz, A.; Martinez-Sanchez, G.; Re, L. Growth factors derived from platelet and its applications in regenerative medicine. Potential use of ozone as activator. Revista Española de Ozonoterapia, v.1, n.1, p.54-73. 2011.

- Silva, R.F. Caracterizações celular e ultra-estrutural do concentrado de plaquetas em cães e gatos e avaliação do seu efeito na osteoartrose em cães. Belo Horizonte, Brasil: Universidade Federal de Minas Gerais, 2012. 88f. Tese (Doutorado em ciência animal).

- Silva, R.F.; Rezende, C.M.F; Paes-Leme, F.O. et al. Evaluación del método del tubo para concentrar plaquetas caninas: estudio celular. Archivos de Medicina Veterinaria, v.43, n.1, p.95-98. 2011a. 
- Silva, R.F; Rezende, C.M.F; Paes-Leme, F.O. et al. Evaluación del método del tubo para concentrar plaquetas felinas: estudio celular. Archivos de Medicina Veterinaria, v.43, n.2, p.187-190. 2011 b.

- Smith, S.E.; Roukis, T.S. Bone and wound healing augmentation with platelet-rich plasma.Clinics in Podiatric Medicine and Surgery, v.26, n.4, p.559-588. 2009.

- Sohn, D.H.; Sokolove, J.; Sharpe, O. et al. Plasma proteins present in osteoarthritic synovial fluid can stimulate cytokine production via Toll-like receptor 4. Arthritis Research and Therapy, v.14, n.1, p.R7. 2012.

- Textor, J. Autologous biologic treatment for equine musculoskeletal injuries: Plateletrich plasma and IL-1 receptor antagonist protein. Veterinary Clinics of North America. Equine Practice, v.27, n.2, p.275-298. 2011.

- Tiwari, M.; Bhargava, R. Platelet rich plasma therapy: a comparative effective therapy with promising results in plantar fasciitis. Journal of Clinics Orthopaedics and Trauma, v.4, n.1, p.31-35. 2013.

- Vagdatli, E.; Gonari, E.; Lazaridou, E. et al. Platelet distribution width: a simple, practical and specific marker of activation of coagulation. Hippokratia, v.14, n.1, p.28$32,2010$.

- Virchenko, O.; Aspenberg, P. How can one platelet injection after tendon injury lead to a stronger tendon after 4 weeks? Interplay between early regeneration and mechanical stimulation. Acta Orthopaedica, v.77, n.5, p.806-812. 2006.

- Wasterlain, A.S.; Braun, H.J.; Dragoo, J.L. Contents and formulations of platelet-rich plasma. Operative Technique in Orthopaedics, v.22, n.1, p.33-42. 2012.

- Weibrich, G; Hansen, T; Kleis, W. et al. Effect of platelet concentration in platelet-rich plasma on peri-implant bone regeneration. Bone, v.34, n.4, p.665-671. 2004.

- Xie, X.; Wu, H. Zhao, S. et al. The effect of platelet-rich plasma on patterns of gene expression in a dog model of anterior cruciate ligament reconstruction. Journal of Surgery Research, v.180, n.1, p.80-88. 2013a.

- Xie, X.; Zhao, S.; Wu, H. et al. Platelet-rich plasma enhances autograft revascularization and reinnervation in a dog model of anterior cruciate ligament reconstruction. Jounal of Surgery Research, v.183, n.1, p.214-222. 2013 b. 
Cómo citar: Osorio-Carmona, E.; de Cássia-Santana, G.; Castro-Varón, J.A.; Alves Ramalho, G.; Guimarães Reis, L.; Jaramillo-Colorado, S.; de Faria Rezende, C.M. Efeito precoce do concentrado autólogo de plaquetas sobre a osteoartrose induzida em coelhos: Níveis plasmáticos de PDGF e TGF- $\beta 1$ e seu potencial como biomarcadores. Revista Veterinaria y Zootecnia, v. 11, n. 1, p. 96-115. DOI: 10.17151/vetzo.2017.11.1.8 